\title{
Derechos de autor sobre bases de datos electrónicas en la legislación guatemalteca
}

\author{
Daniel Herrera \\ Candidato doctoral en Derecho Comercial Internacional \\ Universidad Nacional Chengchi en Taiwán \\ Daniel@Ciencialex.org
}

Fecha de recepción: 16/03/2016 Fecha de aceptación: 13/04/2016

\section{Resumen}

Es indudable que el desarrollo de software es una de las actividades económicas y estratégicas más importantes actualmente. Los datos son centro de cualquier sistema informático y por esta razón las bases de datos y su protección cobran importancia en la economía actual. El presente artículo presenta un análisis de la normativa para protección de bases de datos en Estados Unidos y la Unión Europea para concluir con un análisis de la legislación guatemalteca, todo desde la perspectiva de las bases de datos digitales.

\section{Palabras Clave}

Bases de datos, esquema de base de datos, software, derechos de autor, organización de datos

\begin{abstract}
Software development is one of the most important activities nowadays. Data is at the center of any computerized system and for this reason data bases and their protection are very important in today's economy. This article features an analysis of the regulations for the protection of data bases in the United States and the European Union to conclude with an analysis of Guatemalan legislation, all from the perspective of digital databases.
\end{abstract}

\section{Keywords}

Data bases, data base schema, software, copyright, organization of data. 


\section{Introducción}

Muchas actividades de la vida moderna transcurren entre aplicaciones móviles y servicios proveídos a través de internet. En el año 2011, el peso de actividades relacionadas con internet en la economía de Estados Unidos era ya de 165 billones de dólares USD (OECD 2015). Los datos procesados son el corazón de cualquier software desde los más sofisticados hasta los más sencillos, ya que el propósito de cualquier sistema de información es la obtención, procesamiento e impresión o salida de datos con un fin útil. Dependiendo del software, una de las muchas actividades de la fase de procesamiento, es el almacenamiento permanente de los datos. Los datos que son procesados por un programa de software subyacen en estructuras lógicas que se denominan bases de datos.

Durante mi ejercicio como docente de derecho de la tecnología, he notado que cuestiones relativas a los derechos de autor sobre Bases de Datos Electrónicas son bastante comunes en el medio de los desarrolladores de software, responder de forma exhaustiva a estas cuestiones es el propósito de este artículo.

Es importante señalar que el propósito de este artículo es dilucidar los aspectos de derechos de autor sobre Bases de Datos Electrónicas consideradas en sí mismas, es decir arreglos sistemáticos de datos para un determinado uso, y no sobre sistemas de software gestores de Bases de Datos como por ejemplo SOL Server de Microsoft, Oracle o MySQL, estos son productos de software, propietarios los dos primeros y libre o de código abierto el tercero, cuyos derechos de autor están claramente delineados de acuerdo a sus respectivos regímenes.

\section{2. ¿Que es una Base de Datos?}

La Directiva Europea para la Protección de Bases de Datos, define las bases de datos como "Una colección de trabajos independientes, datos $\mathrm{u}$ otros materiales organizados de forma sistemáti- ca o metódica y accesible de forma electrónica o por otros medios" (Lemley et al., 2006).

Esta definición es lo suficientemente amplia para englobar los distintos elementos y usos de una base de datos por lo que será la que se utilizará en el resto del presente artículo, con la salvedad que se tratará únicamente acerca de las Bases de Datos Electrónicas.

a. Niveles de Abstracción de una Base de Datos: Es útil pensar en una Base de Datos Electrónica desde distintos niveles de abstracción. En un nivel general existen los datos propiamente, los cuales se crean como producto de eventos tales como transacciones comerciales, actos administrativos, acontecimientos deportivos, etc.

Estos datos son susceptibles de ser almacenados como registros, ya sea en una compilación física, como lo sería por ejemplo un directorio telefónico, o en una base de datos electrónica (principal tema del presente artículo).

Como registros en una base de datos electrónica, los datos se pueden manipular, filtrar y representar por medio de filtros e informes generados a través de software. Una base de datos electrónica, es un conjunto de datos organizados de acuerdo a una construcción lógica, diseñada, creada y susceptible de ser consultada y editada a través de software.

Esta estructura lógica establece la forma en que los datos se relacionan con otros y constituye un segundo nivel de abstracción, integrado por el esquema de la base de datos (tablas, campos, claves primarias, claves foráneas y relaciones) y el método elegido para la selección y arreglo de los datos cuando son consultados por los usuarios del sistema (consultas, vistas, informes).

Un último nivel de abstracción sería el constituido por el almacenamiento físico 
de los datos, este almacenamiento no es más que las direcciones físicas en un dispositivo de memoria en que los datos se encuentran (Eaddy, 2008).

b. Elementos de una Base de Datos: Una base de datos es un conjunto de datos organizados de forma sistemática que debido a esta organización pueden presentarse atendiendo a métodos (filtros, vistas e informes) que de forma eficiente transmiten información útil.

Estos elementos, correspondientes al primer y segundo nivel de abstracción mencionados en el apartado anterior, son importantes ya que son estos los que se encuentran protegidos legalmente mediante el sistema de derechos de autor.

- Los datos o información en sí. Los datos son la razón de ser de la base de datos, como se vio antes, sin embargo, estos suelen ser datos del dominio público tales como información fáctica, o trabajos que tienen ya protección como obra literaria de un tercero ajeno a quien compila la base de datos.

En una base de datos de libros por ejemplo, los datos serían los títulos de los libros, los autores, fechas de publicación, etc. Cada uno de estos libros, por supuesto, se encuentra protegido como obra literaria independiente. Por otro lado en una base de datos de la actividad sísmica, los datos serían, fechas y magnitudes de los temblores, los cuales son hechos del dominio público, que no se encuentran protegidos y cualquiera puede compilarlos, su arreglo y selección al compilarlos, sin embargo, sí estarían protegidos (Reed et al., 2011).

- La organización sistemática de los datos: Esta organización sistemática puede entenderse como el esquema relacional que da estructura a los diferentes datos o Esquema de la Base de Datos.

Los Esquemas obedecen al fin utilitario de permitir al software operar los datos de la forma más eficiente, estos esquemas, aunque en principio obedezcan a un fin utilitario pueden tener un valor como expresión creativa separada de sus aspectos puramente funcionales (Eaddy, 2008). Este esquema se puede visualizar mejor en el modelo entidad - relación de la Base de datos.

- Los métodos de selección de la información: Los métodos de selección son criterios utilizados para la selección de los datos que se proveen, estos métodos de organización involucran el esfuerzo y conocimiento de quien compila la base de datos sobre información contextual a los datos, por ejemplo, datos sobre precios de determinada acción pueden existir en el dominio público, sin embargo, seleccionar un conjunto de precios durante determinado periodo que pudieran tener determinado valor predictivo, involucra un considerable esfuerzo y conocimiento, por lo que dicha compilación sobre dichos datos tiene un valor mayor que el conjunto general de datos sobre precios. (Reed et al., 2011). Las vistas y consultas a la base de datos pueden considerarse parte de estos métodos de organización.

\section{Protección de los diferentes elementos de una Base de Datos en Estados Unidos y la Unión Europea}

Antes de entrar a la descripción acerca de los sistemas de protección de las bases de datos en legislaciones con un desarrollo tecnológico superior a Guatemala, como lo son los Estados Unidos y la Unión Europea, cabe una bre- 
ve descripción del distinto trasfondo filosófico en que dichas tradiciones legales sobre propiedad intelectual se han desarrollado.

El derecho continental cercano a una concepción Lockeana de la propiedad ve en el trabajo creativo la propia justificación de la propiedad sobre sus productos. Esta concepción, se justifica con relación a los derechos morales de autor, sin embargo, en materia de derechos económicos deben hacerse otras consideraciones.

El derecho anglosajón mucho más práctico en sus concepciones y con un entendimiento claro de los efectos de introducir conceptos de propiedad sobre el conocimiento, siempre ha basado la introducción de derechos de propiedad intelectual sobre consideraciones económicas (Reed et al., 2011).

a. La Protección de Bases de Datos en Estados Unidos: El debate sobre la protección de las Bases de Datos surge en Estados Unidos casi de forma simultánea al debate sobre la protección de software. Desde los años 50s hasta bien entrada la década del 70, debido a que el desarrollo de la industria del software aún era incipiente, la protección de software y bases de datos era llevada a cabo por medio del sistema de protección al secreto comercial.

Este sistema de protección mediante secreto comercial no ofrecía ninguna garantía contra terceros de buena fe que entraran en conocimiento de un software o base de datos ya divulgados.

A principios de los años ochenta cuando la industria del software había ya madurado y sus productos ya habían devenido en mercancías, la misma industria impulsó una evolución legislativa. Durante esta evolución, se delinearon los contornos de cómo los diferentes sistemas de propiedad intelectual funcionarían en la práctica en la economía digital.
Estos contornos a grandes rasgos son los siguientes, el régimen de patentes protege las ideas innovadoras y el régimen de derechos de autor la expresión creativa, el software en un $90 \%$ cae dentro de esta última categoría, sin embargo, en Estados Unidos existen ciertos casos en que puede beneficiarse con derechos de patente (Lemley et al., 2006) (la conveniencia o no de esto último escapa al alcance de este artículo).

El sustento de la legislación estadounidense relativo a derechos de autor es el artículo primero, parágrafo octavo, cláusula octava de la Constitución de Estados Unidos, el cual permite al Congreso otorgar a los autores propiedad sobre el producto económico de sus creaciones originales.

Esta disposición constitucional se desarrolló a través de varias iteraciones de leyes de derechos de autor (la última de 1976), enmiendas y leyes relacionadas, todas contenidas en el Título 17 del Código de Estados Unidos. ${ }^{1}$

Como es general en el derecho anglosajón, el propósito legislativo es interpretado por las cortes en cada nuevo caso, esta interpretación legislativa tiene fuerza de ley. El trato judicial de las bases de datos, las ha equiparado con las compilaciones reguladas en los parágrafos 101 y 103 del título 17 del Código de Estados Unidos.

"101 ...Una "compilación" es un trabajo formado por la colección o ensamble de materiales preexistentes o de datos seleccionados, coordinados, y arreglados de manera que del trabajo resulte una obra original..."
El Código de Estados Unidos, es un compendio de las Leyes Federales vigentes que está dividido en 52 títulos y se publica cada seis años por la Oficina del Consejo de Revisión Legal de la Cámara de Representantes del Congreso de los Estados Unidos. 
"103 b)...Esta normativa establece que los derechos sobre compilaciones se extiende solo al material contribuido por el autor del trabajo, distinguiéndose de los derechos sobre los derechos existentes sobre el material compilado..."

El caso "leader", como se reconoce en la doctrina anglosajona al caso que establece el criterio judicial aplicable, es el caso de Feist Publications Inc vs Rural Telephone Service Company Inc (1990). Feist publicó un directorio telefónico que incluía determinada información contenida, y muy seguramente obtenida, del directorio publicado por Rural.

Rural entabló demanda, la cual ganó en primera instancia, sin embargo Feist fue capaz de convencer a la corte de apelaciones que la información telefónica no era sujeta a protección como expresión creativa.

El razonamiento de la corte de apelaciones fue el siguiente:

- Las compilaciones únicamente se protegen debido a que son productos intelectuales de su creador.

- La protección garantizada por la ley de Derechos de Autor a las compilaciones se extiende únicamente a los aspectos originales de las mismas, es decir, su selección y arreglo, no los datos fácticos contenidos en las mismas.

- Terceros son libres de copiar los datos, una vez no copien su selección y arreglo los cuales constituyen el esfuerzo material en la creación de una compilación, el "Sudor de la Frente" (Sweat of the Brow) envuelto en crearla (Reed et al., 2011).

Analizando Feist en el marco de nuestra clasificación de los elementos de una base de datos, se puede afirmar que la palabra selección equivale al orden y forma en que una serie de datos son filtrados y presentados, el cual de ser suficientemente original se encuentra protegido, es decir, el diseño de un informe, producto de una base de datos, se encontraría protegido debido al esfuerzo que implica su creación.

El arreglo de los datos puede entenderse como la abstracción lógica u organización sistemática de los datos, el Esquema de la Base de Datos en términos informáticos, no existe criterio judicial claro en cuanto a este punto, sin embargo para tratadistas como Eaddy es bastante claro que los esquemas están protegidos y constituyen el elemento que mayor esfuerzo intelectual requiere para crearse, esta hipótesis sin embargo aún no está sustentada de forma clara en un criterio judicial (Eaddy, 2008).

En cuanto al contenido de las bases de datos, es decir los datos propiamente dichos, la legislación en Estados Unidos no admite su protección ya que constituyen elementos fácticos (Reed et al., 2011), estos sin embargo pueden protegerse como licencias de uso, ejemplo puede ser el catálogo de información bibliográfica de Goodreads u otros similares, dichos catálogos ofrecen licencias gratuitas a sus API (Application Programming Interface) para la creación de aplicaciones por terceros, sin embargo, no permiten que los usuarios copien sus contenidos para crear sus propias bases de datos, la obligación de no copiar los datos deriva del contrato de licencia de usuario y no de regulaciones de Derechos de Autor.

Debido a que la protección contractual en Estados Unidos no es robusta en la protección de contenidos fácticos, existen iniciativas que persiguen proporcionar protección sui generis para bases de datos en Estados Unidos, dicha protección existe en la Unión Europea, como se verá a continuación. 
c. Protección de Bases de Datos en la Unión Europea: La convención de Berna sobre los derechos de autor no menciona de forma expresa a las bases de datos, de forma análoga se ha interpretado que la regulación expresada en el artículo 2, numerales 5 y 6 de dicha convención puede aplicarse a Bases de Datos equiparándolas a las colecciones, que sí se encuentran reguladas.

"5) Las colecciones de obras literarias o artísticas tales como las enciclopedias y antologías que, por la selección o disposición de las materias, constituyan creaciones intelectuales estarán protegidas como tales, sin perjuicio de los derechos de los autores sobre cada una de las obras que forman parte de estas colecciones."

La Directiva Europea número 96/9 introdujo el concepto de la protección sui generis a las bases de datos; este derecho incluye la protección de las bases de datos contra la extracción total o de una parte substancial de sus contenidos, esta protección se otorga cuando ha existido una inversión substancial en su creación y originalidad en la selección y arreglo de sus contenidos, la presencia de estos requerimientos hace que la protección de bases de datos a través de esta normativa sea bastante similar a la estadounidense, con la única diferencia en que incluye provisiones a los contenidos. (Reed et al., 2011)

\section{Protección de los diferentes elementos de una Base de Datos en la legislación guatemalteca}

La Ley de Derechos de Autor y Conexos de Guatemala trata el tema de la protección de bases de datos en sus artículos 16, 21 y fundamentalmente en su artículo 35.

El artículo 16 b) establece que las bases de datos son obras protegidas, el artícu- lo 21 establece lo relativo a los Derechos Económicos o Pecuniarios de Autor y el artículo 35 establece la regulación de las bases de datos como tales.

"Artículo 16. También se consideran obras, sin perjuicio de los derechos de autor sobre las obras originarias, en su caso:

b) Las antologías, diccionarios, compilaciones, bases de datos y similares, cuando la selección o disposición de las materias constituyan una creación original."

"Artículo 21. El derecho pecuniario o patrimonial, confiere al titular del derecho de autor las facultades de utilizar directa y personalmente la obra, de transferir total o parcialmente sus derechos sobre ella y de autorizar cualquiera de los actos siguientes:

vii) El acceso público a bases de datos de ordenadores por medio de telecomunicación; y"

"Artículo 35. Las compilaciones o bases de datos sean que fueren legibles en máquina o en cualquier otra forma, se consideran como colecciones de obras para efectos de su protección de conformidad con esta ley. Esta protección no se extenderá a los datos o material contenido en la compilaciones ni prejuzgará sobre el derecho de autor existente sobre los mismos".

De la legislación se derivan los siguientes puntos:

- Las Bases de Datos están protegidas como colecciones de obras o compilaciones.

- La protección no se extiende a los contenidos de la base de datos solamente se otorga al arreglo y organización de los datos.

- Autorizar el acceso por medio de telecomunicaciones a los contenidos de bases de datos digitales es facultad de los autores. 
Vale la pena analizar la protección de los diferentes elementos de la base de datos atendiendo a la legislación en vigencia.

a. Protección de los Esquemas de Base de Datos: Los esquemas de base de datos no se encuentran protegidos de forma expresa aunque, de acuerdo al artículo 16 , es posible que puedan considerarse dichos esquemas parte de los elementos de "selección o disposición" de los datos que tienen valor original.

b. Protección sobre la Selección de los Datos: La protección sobre las selección de datos que forman parte de la base de datos, se encuentra protegida de forma expresa.

c. Protección sobre los Datos que Integran la Base de Datos: Los datos que integran la base de datos están excluidos expresamente de protección como parte de la base de datos, esto no prejuzga que alguno de estos elementos puede tener protección como obra independiente.

Autorizar el acceso a estos datos en el caso de bases de datos digitales es facultad del autor de la base de datos. Se tiene la facultad de regular el acceso más no el uso de los datos, los cuales están protegidos o no de acuerdo a sus propias características como arriba se menciona, por ejemplo, en el caso de una base de datos de resultados de eventos deportivo, los resultados serían información de dominio público, un arreglo en que estos se encuentran compilados de forma creativa si atraería protección del régimen de derechos de autor.

\section{Conclusiones}

La protección de Bases de Datos Electrónicas se encuentra basada principalmente en dos precedentes, la protección a colecciones y compilaciones y la protección a los programas informáticos, los cuales fueron incluidos en el régimen de derechos de autor en la década de los ochenta.

La protección de Bases de Datos Electrónicas y del Software tanto en la doctrina y legislación europea como la estadounidense se basa en un razonamiento utilitario y económico, esto indudablemente debido a la influencia innegable y pionera de la legislación estadounidense en aspectos de tecnología.

Tomando en cuenta los antecedentes antes mencionados, especialmente la equiparación de las Bases de Datos Electrónicas con las compilaciones y colecciones, es muy fácil argumentar la protección y propiedad sobre las vistas e informes generados de una base de datos, sin embargo, la propiedad sobre esquemas es algo que aún se encuentra en un área gris.

Se recomienda a emprendedores y desarrolladores proteger sus esquemas mediante acuerdos de confidencialidad con la empresa, en el caso de empleados, y a través de cláusulas para prevenir ingeniería inversa en los contratos de licencia de usuario final. También es importante que el acceso a los esquemas se encuentre protegido mediante claves y no esté al alcance de usuarios finales.

En cuento a la protección a los datos propiamente dichos, cabe mencionar que únicamente la Unión Europea cuenta con una regulación en este sentido, por lo que es importante tomar precauciones en las licencias de uso de APIs públicas en el sentido de prohibir que consultas a los datos puedan servir para clonar sus contenidos en servicios competidores.

\section{Referencias}

Eaddy, J. H. (2008). Database Copyright Analysis for Dummies: Protecting Your Intellectual Investment is About the Design, Not the Data. Boston, MA: New England Law Review Vol 42:299 
OECD (2013). Measuring the Internet Economy: A Contribution to the Research Agenda, OECD Digital Economy Papers, No. 226, OECD Publishing.

Lemley, M. A. (2006). Software and Internet law. New York, NY: Wolters Kluwer.
Reed, C., \& Angel, J. (2008). Computer law: the law and regulation of information technology. Oxford: Oxford University Press. 